Revue d'histoire de l'Amérique française

REVUE D.HISTOIRE DE L'AMÉRIQUE FRANÇAISE

\title{
Le rapport agriculture-forêt au Québec : note historiographique
}

\section{Guy Gaudreau}

Volume 33, numéro 1, juin 1979

URI : https://id.erudit.org/iderudit/303752ar

DOI : https://doi.org/10.7202/303752ar

Aller au sommaire du numéro

Éditeur(s)

Institut d'histoire de l'Amérique française

ISSN

0035-2357 (imprimé)

1492-1383 (numérique)

Découvrir la revue

Citer cette note

Gaudreau, G. (1979). Le rapport agriculture-forêt au Québec : note

historiographique. Revue d'histoire de l'Amérique française, 33(1), 67-78.

https://doi.org/10.7202/303752ar d'utilisation que vous pouvez consulter en ligne.

https://apropos.erudit.org/fr/usagers/politique-dutilisation/ 


\section{LE RAPPORT AGRICULTURE-FORÊT AU QUÉBEC: NOTE HISTORIOGRAPHIQUE*}

Guy Gaudreau

L'étude de l'histoire de l'agriculture québécoise dans la perspective du développement du mode de production capitaliste connaît actuellement un intérêt certain ${ }^{1}$. Notre contribution, qui s'inscrit dans cette perspective, s'attache à un aspect spécifique de cette question, soit le rapport agriculture-forêt. C'est d'abord à Alfred Dubuc puis à Normand Séguin que revient le mérite d'avoir mis en évidence la relation structurelle qui noue, ou plutôt nouait, les activités agricoles aux activités forestières dans les régions de colonisation, particulièrement dans la deuxième moitié du $\mathrm{XIX}^{\mathrm{e}}$ siècle $^{2}$. Cette relation se traduit dans la réalité par la vente saisonnière d'une force de travail dont le libre détenteur est encore propriétaire de ses moyens de production (haches, chevaux, etc.); par la vente de denrées agricoles aux chantiers; et enfin par la vente du bois que la terre peut receler (souvent en dépit de la réglementation qui l'interdit aux colons, tant qu'ils n'ont pas obtenu leurs lettres patentes) ${ }^{3}$. Cette re-

* Nous tenons à remercier Messieurs Alfred Dubuc, Paul-André Linteau et Normand Séguin pour leurs remarques opportunes, ainsi que le Centre de recherches et d'innovation urbaines pour son support technique et institutionnel.

1 Citons, par exemple, les ouvrages de Colette Chatillon, L'histoire de l'agriculture au Québec (Montréal, L’Étincelle, 1976); Diane Lessard, L'agriculture et le capitalisme au Québec (Montréal, L’Étincelle, 1976); la revue Anthropologie et Sociétés qui a consacré récemment un numéro à l'agriculture au Québec, 1, 2 (1977).

2 Alfred Dubuc, Les inégalités économiques, Le Devoir, 2 et 3 mars 1973; Normand Séguin, La conquête du sol au XIX e siècle (Québec, Boréal Express, 1977), 31 et «L'économie agro-forestière : genèse du développement au Saguenay au XIX ${ }^{\text {e }}$ siècle», Revue d'histoire de l'Amérique française, 29, 4 (mars 1976) : 559-565.

3 Rappelons que le colon doit satisfaire certaines conditions d'occupation avant d'obtenir ses lettres patentes et de pouvoir exploiter pleinement la matière ligneuse, voir J.-E. Garon, Historique de la colonisation dans la province de Québec de 1826 à 1940 (Québec, 1940), 98-101. 
lation dont la nature, comme nous le verrons, fait l'objet de controverses, demeure un point de repère fondamental pour l'étude du développement du capitalisme dans les régions dites périphériques. C'est pourquoi nous nous proposons de faire non pas une rétrospective systématique, exhaustive et détaillée de tous les auteurs qui ont abordé cette question, mais bien plutôt des principaux porte-parole de chacune de ces thèses.

Grosso modo trois types de relation sont perçus par les historiens et les contemporains quelquefois à l'état pur mais plus souvent avec des nuances, l'un étant privilégié plutôt que les deux autres. Dans le premier type, les producteurs agricoles bénéficient grandement de leur rapport avec l'exploitation forestière, ce qui débouche en quelque sorte sur une relation de complémentarité à l'intérieur de laquelle tout est harmonieux; le second insiste au contraire sur les préjudices subis par l'agriculture, sur le joug qui asservit la production agricole devenue dépendante vis-à-vis de la forêt; enfin, le dernier en est un de concurrence qui aboutit finalement à une relation conflictuelle entre les colons et les marchands de bois.

Les arguments invoqués par ceux qui voient dans cette connexion des deux activités les avantages que retirent les petits producteurs se résument fondamentalement à deux éléments. Installés sur des terres dont la fertilité est souvent plus que douteuse, les agriculteurs sont à l'affût de revenus d'appoint qu'ils trouveront par l'intermédiaire du travail dans les chantiers, ce qui compense les difficultés initiales: De plus, les chantiers servent de débouchés pour l'excédent de leur production, notamment pour l'avoine et la pomme de terre.

Aux colonies qui se sont aventurées sur des terrains de pauvre qualité, elle assure la survie par les débouchés qu'elle offre à leurs produits et le travail qu' elle fournit à leurs habitants. Aussi bien, peut-on dire de la forêt qu'elle est pour l'agriculture un auxiliaire précieux... ${ }^{4}$

Pour Gérald Fortin et Émile Gosselin, cette complémentarité est triple: il y a complémentarité au niveau des revenus, de l'emploi du temps (les deux activités étant bien synchronisées) et enfin des techniques et des outils utilisés.

4 Avila Bédard, «Le milieu forestier», in Esdras Minville, éd., La Forêt (Études sur notre milieu) (Montréal, Fides, 1944), 21. 
...le travail en forêt n'entrait pas en compétition avec le travail agricole. Les opérations forestières se déroulaient du mois de novembre aux mois de janvier ou février. Durant cette période, le travail de la ferme était au ralenti. Les travaux des champs étaient terminés et le soin des animaux était limité au minimum vu que la production laitière s'achevait avec l'automne. Le travail sur la ferme étant ainsi réduit, les enfants et la femme pouvaient en prendre charge. La complémentarité entre l'agriculture et le travail forestier dépassait donc le plan du revenu. ${ }^{5}$

Les deux industries, agriculture et forêt, étaient aussi complémentaires du point de vue des techniques employées. Les outils de base de la coupe étant la hache et le 'bucksaw', instruments qu'on retrouve sur toutes les fermes québécoises. Pour le charriage, le fermier pouvait se servir de ses chevaux de ferme. En fait, le seul investissement nécessaire au fermier qui voulait aller travailler en forêt était le plus souvent un traîneau plus grand et plus fort que celui dont il se servait sur sa ferme. ${ }^{6}$

Signalons que dans cet article les auteurs affirment que les compagnies forestières faisaient en sorte que leurs opérations débutent après la fin des travaux de la ferme ${ }^{7}$. Enfin, dernier élément, la vente du bois que recèlent les lots de ferme est quelquefois signalée sans trop d'insistance par Bédard, qui y voit une autre alternative en vue d'obtenir du numéraire ${ }^{8}$.

Les adeptes de la seconde conception, c'est-à-dire ceux qui insistent sur les inconvénients inhérents au maintien du lien avec l'exploitation forestière, ont d'abord tous reconnu à des degrés divers les avantages que pouvaient en tirer les petits producteurs.

Pour Esdras Minville, la forêt «... est directement nécessaire à la subsistance de la population, et cela à cause du caractère saisonnier de nos grandes exploitations de base: agriculture, pêche, forêt elle-même » ${ }^{9}$. De son côté Raoul Blanchard constate que l'exploitation forestière favorise l'accès aux lots de colonisation, fournit de

5 Gérald Fortin, «Les changements socio-culturels dans une paroisse agricole», in Marcel Rioux et Yves Martin, éds, La société canadienne-française (Montréal, Hurtubise HMH, 1971), 104.

6 Gérald Fortin et Émile Gosselin, «La professionalisation du travail en forêt », Recherches sociographiques, 1, 1 (1960): 34-35.

7 Ibid., 34.

8 Voir Bédard, loc. cit., 21.

329.

9 Esdras Minville, "Le problème social de la forêt», in La Forêt... op. cit., 
futurs colons et génère des revenus d'appoint nécessaires ${ }^{10}$. «Pour le secteur agricole de subsistance, affirme Normand Séguin, le travail en forêt est perçu comme une source indispensable de revenu d'appoint permettant l'achat d'une gamme de produits réputés indispensables ${ }^{11}$. Quant à Gérard Bouchard, il écrit:

... nous pensons que l'activité forestière a pu, dans un court terme, favoriser directement le développement de l'agriculture. D'abord, il est douteux que les chantiers aient généralement entravé les travaux de la terre, étant donné la bonne synchronisation des deux activités ... et à cause de l'abondance de la main-d'œuvre rurale qui permettait de pourvoir simultanément aux besoins de la coupe et de la ferme durant la saison hivernale. En outre, des témoignages de toutes sortes attestent que le revenu forestier était, dans la très grande majorité des cas, entièrement investi dans la ferme. Il a ainsi directement contribué ici et là, et dès avant l'essor de l'industrie des pâtes et papiers, à tirer l'agriculture de l'autosubsistance en l'intégrant davantage au marché. (...) Ailleurs, les bénéfices réalisés dans la vente de produits agricoles aux chantiers ont suscité les mêmes effets. ${ }^{12}$

Cette conception synchronique des deux activités s'oppose totalement à celles de Raoul Blanchard et de Normand Séguin. Le défaut de synchronisation aboutit, selon ceux-ci, à une compression néfaste des activités agricoles, ou plutôt du temps de travail nécessaire pour assurer le bon fonctionnement de l'établissement. Blanchard écrit :

Mais le cultivateur qui fréquente les chantiers part trop tôt à l'automne pour pouvoir faire ses labours: ceux qui vont à la drave écourtent fortement leur saison de printemps; tout le travail de l'exploitation s'en ressent. Le bétail laitier, qui est le bien le plus précieux de la ferme, pâtit de l'absence du maître. ${ }^{13}$

Il y a plus, en prenant goût au travail rémunéré, les «... colons préfèrent souvent le dur, mais lucratif travail dans les chantiers au labeur ingrat et à la rentabilité hypothétique du défrichement d'un lot ${ }^{14}$. Autrement dit, ils «... perdent la mentalité agricole ${ }^{15}$.

10 Raoul Blanchard, Le Canada français (Paris, Fayard, 1960), 132-133.

1 Normand Séguin, "L'Économie agro-forestière..." loc. cit., 560.

12 Gérard Bouchard, «Introduction à l'étude de la société saguenayenne au $\mathrm{XIX}^{\mathrm{e}}$ siècle», Revue d'histoire de l'Amérique française, 31, 1 (juin 1977): 15. op. cit., 50 .

13 Blanchard, op. cit., 134. Voir aussi Normand Séguin, La conquête du sol...,

14 Jean Hamelin et Yves Roby, Histoire économique du Québec, 1851-1896 (Montréal, Fides, 1971), 225. 
Esdras Minville s'exprime ainsi:

... le chantier reste probablement l'institution la plus primitive de notre organisation économique. Son influence sur l'esprit des agriculteurs est nettement pernicieuse. Il faut lui reprocher entre autres - et c'est un reproche très grave - d'avoir introduit dans nos classes rurales l'esprit ouvrier, pis encore, l'esprit prolétaire, c'est-à-dire le goût de la tâche rémunérée à taux convenu et dont on a ni l'initiative ni la responsabilité - esprit qui est à l'opposé même de celui qui doit animer l'agriculteur s'il doit faire un succès de son entreprise. Et c'est une des raisons, peut-être la principale, pour lesquelles dans nos régions miagricoles, mi-forestières, on est justifié de soutenir que l'exploitation forestière tue l'agriculture... ${ }^{16}$

Esdras Minville voit de plus, à cause de la possibilité qu'a le colon, depuis la réglementation de 1849 , d'obtenir un lot à l'intérieur des concessions forestières, un autre aspect préjudiciable à l'établissement d'un colon sur les terres de la Couronne. La réglementation prescrit pour le détenteur d'une concession forestière le droit de faire la coupe du bois selon le diamètre et les essences des arbres. Si un colon obtient un billet de concession à l'intérieur d'une «limite à bois », le propriétaire de cette dernière a jusqu'au $1^{\text {er }}$ mai après l'obtention de ce billet pour exercer ses droits. Il s'ensuit, affirme-t-il, des retards à la colonisation, en raison des pressions exercées par les marchands de bois auprès de l'administration provinciale afin de surseoir à l'attribution des billets de concession.

... en dépit du droit que le gouvernement se réserve expressément de retraire (sic) pour fin de colonisation toute partie des forêts affermées chacun sait que la colonisation doit subir de longs retards, juste assez longs souvent pour permettre aux concessionnaires de dépouiller de leur bois les terres à coloniser. ${ }^{17}$

Pour Normand Séguin «... l'étude des relations entre les activités agricoles et celles de la forêt permet de déceler un cas spécifique de développement du sous-développement ... ${ }^{18}$, c'est-à-dire

\footnotetext{
15 Blanchard, op. cit., 134.

16 Esdras Minville, «Le problème social...", loc. cit., 331.

Esdras Minville, "La colonisation», L'Actualité économique, XVIII (2 mai

18 Normand Séguin, La conquête du sol..., op. cit., 32.
} 1942): 139 
«... la marginalisation d'une partie de l'agriculture et son maintien dans une forme dégradée» ${ }^{19}$. Le processus par lequel l'activité agricole demeure non-développée, en stagnation et au niveau de la semisubsistance peut se résumer brièvement. Sous l'effet de la demande internationale, l'exploitation forestière pré-industrielle caractérisée par la prépondérance des activités de circulation et du capital variable, se déplace entraînant dans son sillon l'agriculture de subsistance vers les régions périphériques dont l'attrait réside dans la possibilité de soutirer un revenu d'appoint. "Toutefois, cette extension dans l'espace de l'agriculture n'entraîna pas une amélioration qualitative de cette activité (...) Au contraire, la nouvelle population agricole ... s'enracina dans la subsistance. ${ }^{20}$ Trois facteurs sont invoqués: 1) les colons s'installent conséquemment sur des sols trop pauvres, 2) à l'extérieur d'un marché régional qui aurait pu servir de débouchés pour l'excédent de la production, 3) et saisissent les occasions de travail dans les chantiers, le transport et le flottage du bois, réduisant ainsi le temps nécessaire à l'exploitation de la ferme ${ }^{21}$. En somme, l'agriculture est dominée et demeure dans un état de dépendance vis-à-vis l'exploitation forestière qui lui impose son rythme.

Gérard Bouchard, en formulant ses critiques contre la thèse de Séguin, adopte une interprétation nuancée. À court terme, l'exploitation forestière «a favorisé un démarrage agraire.» $\grave{A}$ long terme, dit-il, cette hypothèse ne demeure pas valable, «... le bois s'avère au total un très médiocre démarreur pour la société rurale. Encore que cette carence n'agisse que très indirectement sur l'agriculture: ce sont en effet les villes qui dans l'ensemble ont le plus directement souffert de l'économie du bois, celle-ci inhibant leur développement ... et privant l'agriculture d'un marché local par ricochet ${ }^{22}$. L'absence d'un marché régional devient la cause première du dépérissement des activités agricoles. "Le revenu de la coupe atténuait la pauvreté, il ne la créait pas» ${ }^{23}$. En fait, un seul élément peut être retenu contre la coupe; c'est le fait que l'exploitation forestière, utilisant les voies d'accès naturelles, a favorisé très tardivement la mise en place de voies de communication.

\footnotetext{
19 Ibid., 34.

20 Idem, «L'économie agro-forestière..., "loc. cit., 563.

21 Voir La conquête du sol..., op. cit., 36.

22 Bouchard, loc. cit., 15.

23 Ibid., 16.
} 
Pour les tenants de la thèse conflictuelle, la relation concurrentielle entre ces deux activités a pour fondement le sol à coloniser et la ressource qui s'y trouve, c'est-à-dire le bois. Un conflit éclate très souvent lorsque le colon et le marchand de bois se disputent l'acquisition des mêmes terres publiques ${ }^{24}$.

La rivalité qui en résulte est amplifiée par les droits de propriété simultanés que possèdent colon et marchand de bois sur un lot de colonisation situé à l'intérieur d'une concession forestière.

Il est très malheureux qu'il existe un état de chose qui fasse du colon l'ennemi du marchand de bois et vice versa. Le mal vient du fait que, d'après nos lois existantes, le colon et le marchand de bois ont des droits de propriété simultanés... Il serait absurde de mettre en conflit des droits concurrents. Dans ce cas, c'est le plus faible qui est la victime du plus fort. ${ }^{25}$

A.R.M. Lower demeure à notre connaissance un des seuls historiens qui ait insisté de manière significative sur cette interprétation, qui, pourtant, se retrouve fréquemment chez les contemporains du tournant du siècle ${ }^{26}$. Jusqu'au milieu du $\mathrm{XIX}^{\mathrm{e}}$ siècle, les colons et les marchands de bois sont en bons termes, mais à partir de ce moment »... (a) clash of interests had occured which was destined to prove the chief problem in the administration of the frontier districts $"{ }^{27}$. Un conflit au niveau de l'utilisation du domaine public apparaît au fur et à mesure que progresse la colonisation. Comme il le rappelle: "When he (the lumberman) saw his precious pines going up in smoke to make a miserable mountain farm, and the rocks or sands on which they has stood laid bare, he was quick to cry out $» 28$.

24 Voir J.-C. K. Laflamme, La colonisation et la Forêt, conférence faite à l'Université Laval le 18 mars 1908 et publiée dans Convention Forestière Canadienne par le département des Terres et Forêts (Québec, 1908), 8.

25 Henri Bourassa, (témoignage), Rapport de la commission de la colonisation de la province de Québec, Enquêtes à Montréal (Québec, Charles Pageau, 1904), 315. Voir aussi, Joseph Levitt, Henri Bourassa and the golden calf, 1900-1914 (Ottawa, les Éditions de l'Université Laval, 1972), 62-63.

26 A.R.M. Lower, "The Assault on the Laurentian: Barrier, 1850-1870", The Canadian Historical Review, X (1929): 294-307 et "Settlement and the forest frontier in Eastern Canada in W. A. MacKingtosh et W.L.G. Joerg, éditeurs, Canadian Frontier of settlement, IX (Toronto, Macmillan, 1936) (voir notamment le chapitre V intitulé: Lumbering and farming: rivals on the laurentian plateau).

27 Idem, "Settlement and the forest... ", loc. cit., 48.

28 Idem, "The Assault...", loc. cit., 295-296. 
Finalement, le colon, dont Lower fait trois catégories, nuit de deux manières au marchand de bois: le colon utilise à son profit le bois de son lot, réduisant ainsi le potentiel forestier de sa concession ou plus simplement encore y met «accidentellement»le feu.

There was first of all the individual settler. He probably would be a person who had been working in the lumber camps in the winters and had found some favourable meadow land nearby the camps. It was easy to get a Crown grant, and as long as the camps remained in the neighbourhood, all went well.

There was another class of settler to whom as clean a bill of health was not be given. This was the man who took out a grant, no with any idea of clearing and farming, by simply in order to get possession of the timber upon it. The system of payment for grants of Crown land was in use by this time, but, as payment could be made in a number of annual instalments, it was profitable to "settle", pay one instalment, cutt of and sell the timber, and then move on. (...)

The third class of person to invade the Barrier was the squatter. He had no legal rights and was not encouraged, for he was a nuisance and a danger: a danger because of the likelihood of forest fires araising from his irresponsible clearings. He was not a major element in the assault. ${ }^{29}$

Afin de dépasser le niveau descriptif, il devient maintenant important d'aligner les points de divergences, les contradictions entre les trois thèses mais aussi entre les raisonnements. Ces contradictions apparaissent de prime abord nettement irréconciliables; cependant, cette constatation déroutante fait ressortir finalement un objet de recherche et d'analyse très riche pour l'historien. À la limite, le questionnement amorcé par celles-ci est double: comment réconcilier les préjudices sérieux que subit l'agriculteur avec les avantages qu'il retire et comment mettre en relation une conception harmonieuse des rapports sociaux avec une perception conflictuelle? Les deux questions sont foncièrement différentes et font appel à des registres distincts.

D'abord les effets générés par ce lien structurel sur l'agriculture sont jugés par certains auteurs en fonction d'une représentation idéologique de la place que doivent occuper les activités agricoles et de leur nature. Dans le cas d'Esdras Minville, cette remarque s'applique totalement. Rappelons ses mots: "Il faut lui reprocher entre autres - et c'est un reproche très grave - d'avoir introduit 
dans nos classes rurales l'esprit ouvrier, pis encore, l'esprit prolétaire...». Minville se fait le porte-parole de l'idéologie agrarienne tout comme Raoul Blanchard d'ailleurs. C'est dans cette perspective qu'est organisée leur argumentation. À l'opposé, Avila Bédard, ancien sous-ministre au Ministère des Terres et Forêts, c'est-à-dire préposé à l'antichambre des «Lumber Lords», véhicule l'idéologie du développement où la création d'emplois salariés et l'exploitation des ressources naturelles deviennent une marotte ${ }^{30}$. Il ne peut finalement qu'admettre les bienfaits de l'exploitation forestière pour l'agriculture.

Il devient révélateur dans ce contexte de soulever la divergence, chez les auteurs, à propos de la synchronisation des emplois du temps. On voit bien ce qu'on désire voir, d'autant plus qu'il est fort probable que, si les colons qui ne participaient qu'au travail dans les chantiers n'écourtaient point leur temps de travail nécessaire comme producteurs agricoles, ceux qui en plus devenaient draveurs ou charroyeurs réduisaient l'ampleur ou la durée de leurs travaux printaniers.

Gérald Fortin, Émile Gosselin, Normand Séguin et Gérard Bouchard se démarquent nettement de ce courant idéologique. En désaccord sur l'importance du rôle de l'exploitation forestière et sur les biais (directs ou indirects) par lesquels l'exploitation forestière nuit à l'agriculture, désaccord qui devient quant à nous secondaire, ces deux derniers conservent néanmoins un point de référence commun: l'agriculture se «sous-développe", se maintient dans une forme de semi-subsistance, dans un état «d'urgence» lorsqu'elle est comparée à l'agriculture du centre du Québec qui se convertit lentement en fonction des marchés.

En réalité, on découvre par l'exemple du Saguenay que le déplacement des activités forestières vers la périphérie a provoqué la migration de l'agriculture de subsistance dans cette même direction au moment où, au centre du Québec, s'amorçait une lente reconversion de l'espace agraire en fonction des marchés. ${ }^{31}$

Ce maintien d'une agriculture dégradée, comme le rappelle l'auteur de La conquête du sol au XIX ${ }^{e}$ siècle, assure un flux abondant de main-d'œuvre pour l'abattage et le transport du bois, main1907.

30 Voir à titre indicatif le rapport du ministre des Terres et Forêts pour l'année

31 Normand Séguin, «L'économie agro-forestière...", loc. cit., 562. 
d'œuvre d'ailleurs à bon marché puisqu'une partie des moyens de subsistance nécessaires à la reproduction de la force de travail est tirée de la production agricole. Cela nous amène en fin de compte à introduire la deuxième partie de notre questionnement dans la mesure où cette dépendance de l'agriculture (Séguin) qui donne des «signes d'affranchissement» (Bouchard) ou encore cette complémentarité agriculture-forêt (Fortin et Gosselin) laissent somme toute, peu de place à des rapports concurrentiels et conflictuels ${ }^{32}$.

Une constatation s'impose de prime abord: ni Normand Séguin, ni Gérard Bouchard n'ont intégré dans leurs analyses la vente du bois par le colon; tout tourne autour du travail en forêt et de façon secondaire autour de la vente de l'excédent de la production agricole. En dépit du fait que ses propos peuvent s'interpréter comme une critique mal dissimulée contre les colons qui «nuisent» aux exploitants forestiers il renverse ainsi complètement le rapport: ce n'est plus l'agriculture qui subit des préjudices des activités forestières, mais l'inverse, A.R.M. Lower conserve quand même le mérite de rappeler cette dimension conflictuelle. Cependant, reste à savoir quelle est l'importance de cette vente de bois?

Les données statistiques concernant cette vente font défaut et ce, d'autant plus que les colons agissaient le plus souvent dans l'illégalité, donc n'avaient pas intérêt à divulguer leur production. Certains indices, cependant, tendent à démontrer que ce commerce était important. Les rapports annuels du commissaire des Terres de la Couronne ou du ministre des Terres et Forêts fourmillent littéralement de réflexions concernant les prétendus colons qui, non seulement enfreignent la loi mais, en plus, inondent le marché de produits pas toujours de bonne qualité. Dans son rapport de 1908, le commissaire présente des données qui accordent un poids considérable, et probablement exagéré, à ce commerce.

...il a été transporté (en 1908) aux États-Unis pour là être converties en pâtes à papier, 720639 cordes de bois de pulpe, et que d'après nos données, le bois provenant des Terres de la Couronne, sous permis de coupe ne figure là-dessus que pour une quantité de 147159 cordes. ${ }^{33}$

32 Il faut imputer partiellement cette absence à l'insuffisance de données; c'est le cas notamment pour Normand Séguin qui voit bien les rivalités concernant le contrôle ou l'acquisition des ressources au moment où le secteur des pâtes et papiers connaît un développement. Voir La conquête du sol..., op. cit., 132-142. 1980, IX-X.

33 Rapport du ministre des Terres et Forêts pour l'année finissant le 30 juin 
Un tableau de l'Annuaire Statistique du Québec de 1915 semble confirmer que les agriculteurs, dont font partie les colons, occupent une place non négligeable dans cette production. On dénombre pour l'année 1914 en billots, 759664238 pieds mesure de planche provenant des lots privés contre 938493344 PMP provenant des concessions forestières ${ }^{34}$. Ces données ponctuelles, qui recouvrent la production des grands propriétaires fonciers et des petits producteurs, demeurent des indices approximatifs mais suffisants.

En terminant ce survol historiographique, nous serions tenté d'apporter l'hypothèse suivante: deux tendances contradictoires déterminent la situation des agriculteurs dans les régions de colonisation. Sous l'effet de la pénétration du capitalisme, une tendance à la prolétarisation très longtemps saisonnière s'exerce chez les colons pour qui la recherche de numéraire est une nécessité absolue; en même temps, cette pénétration du capitalisme, en reconnaissant socialement une valeur d'échange à la matière ligneuse, incite le colon (particulièrement avec l'essor des pâtes et papiers) à exploiter le bois qu'il trouvera éventuellement sur son lot ou à proximité. Cette hypothèse, qui a le mérite d'intégrer théoriquement le colon de bonne foi et le prétendu colon, et mieux encore d'expliquer les divergences et les contradictions apparues dans l'historiographie, permet de reconstituer un développement équivoque du capitalisme dans la mesure où le développement des forces productives dans les scieries et usines de pâtes et papiers, en commandant un approvisionnement sans cesse accru de matières ligneuses, dont la coupe restera longtemps artisanale, a suscité une production de bois. Les terres de la Couronne étant doublement concédées, c'est-à-dire à l'exploitant forestier (la concession forestière) et au colon (le billet de concession), cette production fera l'objet, de la part de l'État, d'une législation sévère en vue de la restreindre, mais d'un contrôle peu efficace.

C'est dans cette perspective que nous entreprenons un programme de recherche sur les politiques forestières et de colonisation au Québec de 1840 au début du $\mathrm{XX}^{\mathrm{e}}$ siècle. Les législations et les règlements analysés à travers un dépouillement plus large des publications officielles (rapports annuels des commissaires, rapports de commissions d'enquêtes, réponses aux adresses, etc...) définissent une documentation très riche pour l'étude des régions de colonisation et

34 Annuaire statistique du Québec (1915): 476. 
du thème agro-forestier. Cependant, cette documentation ne lève pas totalement le voile, ni cette note d'ailleurs. L'angle d'observation privilégie une dimension parmi d'autres: la lutte pour l'acquisition du sol et de ses ressources sur les terres publiques dont l'État est le gestionnaire partial. 